\title{
Flow of Generalized Burgers' Fluid Induced by Sawtooth Pulses Stress with Quadratic Edges
}

\author{
Qamar Sultan1, Zaib Un Nisa², Mudassar Nazar1,2* \\ ${ }^{1}$ Centre for Advanced Studies in Pure and Applied Mathematics, Bahauddin Zakariya University, Multan, Pakistan \\ ${ }^{2}$ School of Mathematical Sciences, University of Science and Technology of China, Hefei, China \\ Email: *mudassar_666@yahoo.com
}

How to cite this paper: Sultan, Q., Nisa, Z.U. and Nazar, M. (2018) Flow of Generalized Burgers' Fluid Induced by Sawtooth Pulses Stress with Quadratic Edges. Journal of Applied Mathematics and Physics, 6, 1258-1277.

https://doi.org/10.4236/jamp.2018.66106

Received: April 8, 2018

Accepted: June 23, 2018

Published: June 26, 2018

Copyright $\odot 2018$ by authors and Scientific Research Publishing Inc. This work is licensed under the Creative Commons Attribution International License (CC BY 4.0).

http://creativecommons.org/licenses/by/4.0/

\begin{abstract}
This paper presents the unsteady magnetohydrodynamic (MHD) flow of a generalized Burgers' fluid between two side walls perpendicular to a plate. The flow is generated from rest at time $t=0^{+}$induced by stress applied on the bottom plate. The stress is assumed to be like sawtooth pulses with quadratic edges. The solutions obtained by means of the Laplace, and Fourier cosine and sine transforms are presented as a sum between the corresponding Newtonian and non-Newtonian contributions. Graphs are sketched for various parameters of interest.
\end{abstract}

\section{Keywords}

Generalized Burgers' Fluid, Sawtooth Pulses Stress, Fourier Cosine and Sine Transforms

\section{Introduction}

The research in the field of non-Newtonian fluid motion has considerably grown because of the wide range of their applications in different fields. Such type of flows are of interest to workers in cement industry, medicine and geology, e.g., in dams, clay rotation, artificial surfing, heartbeat, motion in the liquid core of the earth during earthquakes. Among them the Burgers' fluid as a special non-Newtonian fluid got special attention in describing polymeric liquids. There is a lot of work published on non-Newtonian fluid models but we mention here the references that are mostly related to our work.

Zheng et al. [1] obtained the exact analytical solutions for generalized Maxwell fluid over an infinite flat plate due to oscillatory and constantly accelerating motion of plate. Expressions for the velocity field and the corresponding shear stress are expressed in series forms in terms of generalized G and R. Ghosh and 
Sana [2] solved an initial value problem designed for the motion of an Oldroyd-B fluid bounded by an infinite rigid non-conducting plate. The unsteady flow was generated from rest in the fluid due to velocity sawtooth pulses subjected on the plate in the presence of an external magnetic field. Akhtar et al. [3] and Fetecau et al. [4] determined the velocity field and the adequate tangential stresses corresponding to the unsteady flows of Maxwell and Oldroyd-B fluids, respectively, induced by a constantly accelerating plate between two side walls perpendicular to the plate by means of Fourier sine transform. Chakraborty and Ray [5] investigated the Couette flow between two parallel walls when one of the walls was set into motion by random pulses in the presence of magnetic field. Makar [6] presented the solution for MHD flow between two parallel plates when one of the plates was subjected to velocity tooth pulses, neglecting the induced magnetic field. Khan and Zeeshan [7] studied the magneto hydrodynamic flow of an Oldroyd-B fluid through over a plate. The motion was generated in the fluid due to the velocity sawtooth pulses applied on the plate.

In all of the above citations, the conditions on the boundary are given in terms of velocity. The stress at the boundary gives important information about the nature of dissipation at the boundary. A little work in the literature is available where stress is given on the boundary. Sohail et al. [8] provided starting solutions in close-form corresponding to the unsteady motion of a Maxwell fluid between two parallel walls due to an infinite plate that applies oscillating shear stresses to the fluid. Ghosh and Sana [9] provided the unsteady motion of an Oldroyd-B fluid bounded by two infinite parallel plates in presence of an external magnetic field. The flow is supposed to generate impulsively from the rest induced by rectified sine pulses applied periodically on the upper plate. Jamil et al. [10] and Shahid et al. [11] determined the starting solutions for the motions of Oldroyd-B fluids induced by quadratic, and cosine and sine oscillating time dependent shear stress, respectively. Sultan et al. [12] analyzed the unsteady flow of a Generalized Burgers' fluid between two parallel walls perpendicular to a plate in the presence of magnetic field. The fluid was set into motion induced by the rectified sine pulses shear stress. Some recent attempts involving oscillating motions induced by stress have been made in many studies [13] [14] [15] [16] [17].

The objective of the present work is to study the magnetohydrodynamic flow of a Generalized Burgers' fluid between two parallel side walls perpendicular to a plate. The flow is generated by the sawtooths with quadratic edges shear stress. The MHD flow involving such fluids has promising applications on the development of energy generation MHD pumps. The boundary condition used is of interest as sound waves, light waves, and ocean waves travel like the form of sawtooth pulses. Study of sawtooth pulses flows is also important because of their increasing applications in aerospace science, astrophysics, atmospheric science, and physiological fluid dynamics as by Ghosh [18]. Moreover, a sawtooth wave has strong electromagnetic properties. A sawtooth pulse may be used in the detection of magnetic effect on electrically conducting flows of biological 
fluids. Analytical expressions for the velocity field and the shear stresses are determined by means of the Laplace transform, Fourier cosine, and sine transforms. Finally, graphs are plotted using a numerical procedure for the inverse Laplace transform namely the Stehfest's algorithm [19] to illustrate the influence of the parameters involved.

\section{Governing Equations}

We consider the unsteady flow of an incompressible generalized Burgers' fluid at rest over an infinite flat plate in the $(x, y)$-plane, and between two parallel side walls separated by a distance $d$ apart, situated in the planes $y=0$ and $y=d$. The $\mathrm{z}$-axis is taken in the direction of flow. Initially, the system is at rest. At time $t=0^{+}$, the plate applies a pulsating shear to the fluid induced by sawtooth pulses with quadratic edges.

If the fluid is at rest up to the moment $t=0$, then

$$
V(x, y, 0)=0, S(x, y, 0)=\frac{\partial S(x, y, 0)}{\partial t}=0 .
$$

The velocity field $V$ and stress $S$ are of the form

$$
V=V(x, y, t)=w(x, y, t) \hat{k}, S=S(x, y, t),
$$

and the meaningful governing equations are

$$
\begin{gathered}
\left(1+\lambda_{1} \frac{\partial}{\partial t}+\lambda_{2} \frac{\partial^{2}}{\partial t^{2}}\right) \tau_{1}(x, y, t) \\
=\mu\left(1+\lambda_{3} \frac{\partial}{\partial t}+\lambda_{4} \frac{\partial^{2}}{\partial t^{2}}\right) \frac{\partial w(x, y, t)}{\partial x} \text { for } \tau_{1}(x, y, 0)=0 . \\
\left(1+\lambda_{1} \frac{\partial}{\partial t}+\lambda_{2} \frac{\partial^{2}}{\partial t^{2}}\right) \tau_{2}(x, y, t) \\
=\mu\left(1+\lambda_{3} \frac{\partial}{\partial t}+\lambda_{4} \frac{\partial^{2}}{\partial t^{2}}\right) \frac{\partial w(x, y, t)}{\partial y} \text { for } \tau_{2}(x, y, 0)=0 . \\
\left(1+\lambda_{1} \frac{\partial}{\partial t}+\lambda_{2} \frac{\partial^{2}}{\partial t^{2}}\right) \frac{\partial w(x, y, t)}{\partial t} \\
=v\left(1+\lambda_{3} \frac{\partial}{\partial t}+\lambda_{4} \frac{\partial^{2}}{\partial t^{2}}\right)\left(\frac{\partial^{2}}{\partial x^{2}}+\frac{\partial^{2}}{\partial y^{2}}\right) w(x, y, t) \\
-\frac{\sigma \beta_{0}^{2}}{\rho}\left(1+\lambda_{1} \frac{\partial}{\partial t}+\lambda_{2} \frac{\partial^{2}}{\partial t^{2}}\right) w(x, y, t)-\frac{v \phi}{k}\left(1+\lambda_{3} \frac{\partial}{\partial t}+\lambda_{4} \frac{\partial^{2}}{\partial t^{2}}\right) w(x, y, t) .
\end{gathered}
$$

where $\lambda_{1}$ and $\lambda_{2}$ are the relaxation and the retardation times, $\lambda_{3}$ and $\lambda_{4}$ are the material constants, $\tau_{1}=S_{x z}(x, y, t)$ and $\tau_{2}=S_{y z}(x, y, t)$ are the nontrivial shear stresses, $\mu$ is dynamic viscosity, $v$ is kinematic viscosity, $\sigma$ is electrical conductivity, $\beta_{0}$ is strength of applied Magnetic field, $\rho$ is density, $\phi$ is porosity of medium, and $k$ permeability of the medium.

We use the following appropriate initial conditions

$$
w(x, y, 0)=\frac{\partial w(x, y, 0)}{\partial t}=\frac{\partial^{2} w(x, y, 0)}{\partial t^{2}}=0, \text { for } x>0, y \in[0, d],
$$


the boundary conditions

$$
\begin{gathered}
\left.\left(1+\lambda_{1} \frac{\partial}{\partial t}+\lambda_{1} \frac{\partial^{2}}{\partial t^{2}}\right) \tau_{1}(x, y, t)\right|_{x=0} \\
=\left.\mu\left(1+\lambda_{3} \frac{\partial}{\partial t}+\lambda_{4} \frac{\partial^{2}}{\partial t^{2}}\right) \frac{\partial w(x, y, t)}{\partial x}\right|_{x=0}=U f(t) \text { for } y \in(0, d) \text { and } t>0, \\
w(x, 0, t)=w(x, d, t)=0, \text { for } x, t>0,
\end{gathered}
$$

and the natural conditions

$$
w(x, y, t)=\frac{\partial w(x, y, t)}{\partial x} \rightarrow 0 \text { as } x \rightarrow \infty, y \in[0, d], t>0 .
$$

Introducing the following nondimensional relations

$$
\begin{aligned}
& \left\{w^{*}=\frac{w}{U}, x^{*}=\frac{x}{d}, y^{*}=\frac{w}{d}, z^{*}=\frac{z}{d}, t^{*}=\frac{t \mu}{\rho d^{2}}, \lambda_{1}^{*}=\frac{\lambda_{1} v}{d^{2}},\right. \\
& \left.\lambda_{2}^{*}=\frac{\lambda_{2} v^{2}}{d^{4}}, \lambda_{3}^{*}=\frac{\lambda_{3} v}{d^{2}}, \lambda_{4}^{*}=\frac{\lambda_{4} v^{2}}{d^{4}}, \tau_{1}=\frac{d S_{x z}}{\mu U}, \tau_{1}=\frac{d S_{x z}}{\mu U}\right\}
\end{aligned}
$$

Equations (3)-(5) in dimensionless form, after removing the sign *, become

$$
\begin{gathered}
\left(1+\lambda_{1} \frac{\partial}{\partial t}+\lambda_{2} \frac{\partial^{2}}{\partial t^{2}}\right) \tau_{1}(x, y, t) \\
=\left(1+\lambda_{3} \frac{\partial}{\partial t}+\lambda_{4} \frac{\partial^{2}}{\partial t^{2}}\right) \frac{\partial w(x, y, t)}{\partial x} \text { for } \tau_{1}(x, y, 0)=0 \\
\left(1+\lambda_{1} \frac{\partial}{\partial t}+\lambda_{2} \frac{\partial^{2}}{\partial t^{2}}\right) \tau_{2}(x, y, t) \\
=\left(1+\lambda_{3} \frac{\partial}{\partial t}+\lambda_{4} \frac{\partial^{2}}{\partial t^{2}}\right) \frac{\partial w(x, y, t)}{\partial y} \text { for } \tau_{2}(x, y, 0)=0 \\
\left(1+\lambda_{1} \frac{\partial}{\partial t}+\lambda_{2} \frac{\partial^{2}}{\partial t^{2}}\right) \frac{\partial w(x, y, t)}{\partial t} \\
=\left(1+\lambda_{3} \frac{\partial}{\partial t}+\lambda_{4} \frac{\partial^{2}}{\partial t^{2}}\right)\left(\frac{\partial^{2}}{\partial x^{2}}+\frac{\partial^{2}}{\partial y^{2}}\right) w(x, y, t) \\
-\Omega\left(1+\lambda_{1} \frac{\partial}{\partial t}+\lambda_{2} \frac{\partial^{2}}{\partial t^{2}}\right) w(x, y, t)-\varepsilon\left(1+\lambda_{3} \frac{\partial}{\partial t}+\lambda_{4} \frac{\partial^{2}}{\partial t^{2}}\right) w(x, y, t) .
\end{gathered}
$$

where $\Omega=\frac{\sigma \beta_{0}^{2} d^{2}}{\rho v}$ is the dimensionless magnetic parameter and $\varepsilon=\frac{\phi d^{2}}{k}$ is the dimensionless porosity parameter.

While the initial, boundary and natural conditions in dimensionless form become

$$
\begin{gathered}
w(x, y, 0)=\frac{\partial w(x, y, 0)}{\partial t}=\frac{\partial^{2} w(x, y, 0)}{\partial t^{2}}=0, \text { for } x>0, y \in[0,1], \\
\left.\left(1+\lambda_{1} \frac{\partial}{\partial t}+\lambda_{1} \frac{\partial^{2}}{\partial t^{2}}\right) \tau_{1}(x, y, t)\right|_{x=0} \\
=\left.\left(1+\lambda_{3} \frac{\partial}{\partial t}+\lambda_{4} \frac{\partial^{2}}{\partial t^{2}}\right) \frac{\partial w(x, y, t)}{\partial x}\right|_{x=0}=f(t) \text { for } y \in(0,1) \text { and } t>0,
\end{gathered}
$$




$$
\begin{gathered}
w(x, 0, t)=w(x, 1, t)=0 \text { for } x, t>0, \\
w(x, y, t)=\frac{\partial w(x, y, t)}{\partial x} \rightarrow 0 \text { as } x \rightarrow \infty, y \in[0, d], t>0 .
\end{gathered}
$$

According to the nature of the applied stress, we assume that the mathematical form of the function $f(t)$ is

$$
f(t)=\frac{1}{T^{2}}\left(t^{2} H(t)+2 \sum_{p=1}^{\infty}(-1)^{p}(t-p T)^{2} H_{p T}(t)\right),
$$

where $H(\cdot)$ is defined as $H_{p T}(t)=0$ for $t \leq p T$ and $H_{p T}(t)=1$ for $t>p T$.

In order to solve the problem, we use the Laplace transform technique and Fourier cosine and sine transforms in this order.

\section{Calculation of the Velocity Field}

Applying the Laplace transform to Equation (13), we obtain the following problem

$$
\begin{aligned}
& \left(1+\lambda_{1} q+\lambda_{2} q^{2}\right) q \bar{w}(x, y, q) \\
& =\left(1+\lambda_{3} q+\lambda_{4} q^{2}\right)\left(\frac{\partial^{2}}{\partial x^{2}}+\frac{\partial^{2}}{\partial y^{2}}\right) \bar{w}(x, y, q) \\
& -\Omega\left(1+\lambda_{1} q+\lambda_{2} q^{2}\right) \bar{w}(x, y, q)-\varepsilon\left(1+\lambda_{3} q+\lambda_{4} q^{2}\right) \bar{w}(x, y, q) .
\end{aligned}
$$

The Laplace transform $\bar{w}(x, y, q)$ of the function $w(x, y, t)$ has to satisfy the conditions

$$
\begin{gathered}
\left.\left(1+\lambda_{1} q+\lambda_{2} q^{2}\right) \bar{\tau}_{1}(x, y, q)\right|_{x=0}=\left.\left(1+\lambda_{3} q+\lambda_{4} q^{2}\right) \frac{\partial \bar{w}(x, y, q)}{\partial x}\right|_{x=0} \\
=\frac{2}{T^{2}}\left(\frac{1}{q^{3}}+2 \sum_{p=1}^{\infty}(-1)^{p} \exp (-p T q) \frac{1}{q^{3}}\right), \text { for } y \in[0,1] \\
w(x, 0, q)=w(x, 1, q)=0, \text { for } x>0 \\
\bar{w}(x, y, q)=\frac{\partial \bar{w}(x, y, q)}{\partial x} \rightarrow 0 \text { as } x \rightarrow \infty, y \in(0, d) .
\end{gathered}
$$

Multiplying both sides of Equation (19) by $\sqrt{\frac{2}{\pi}} \cos (\zeta x) \sin \left(\lambda_{n} y\right)$, where $\lambda_{n}=n \pi$, integrating with respect to $x$ and $y$ from 0 to $\infty$ and 0 to 1 respectively, and bearing in mind the Equations (20)-(22), we find that

$$
\begin{aligned}
& \bar{w}_{n}(\zeta, q)=2 \sqrt{\frac{2}{\pi}} \frac{1}{T^{2}} \frac{(-1)^{n}-1}{\lambda_{n}} \\
& \times \frac{1}{\lambda_{2} q^{3}+\left(\lambda_{1}+\lambda_{4}\left(\zeta^{2}+\lambda_{n}^{2}\right)+\lambda_{2} \Omega+\lambda_{4} \varepsilon\right) q^{2}+\left(1+\lambda_{3}\left(\zeta^{2}+\lambda_{n}^{2}\right)+\lambda_{1} \Omega+\lambda_{3} \varepsilon\right) q+\left(\zeta^{2}+\lambda_{n}^{2}\right)+\Omega+\varepsilon} \\
& \times \frac{1}{q^{3}}\left(1+2 \sum_{p=1}^{\infty}(-1)^{p} \exp (-p T q)\right)
\end{aligned}
$$


where

$$
\bar{w}_{n}(\zeta, q)=\sqrt{\frac{2}{\pi}} \int_{0}^{\infty} \int_{0}^{1} \bar{w}(x, y, q) \cos (\zeta x) \sin \left(\lambda_{n} y\right) \mathrm{d} y \mathrm{~d} x ; n=1,2,3, \cdots
$$

Equation (23) can also be written as

$$
\begin{aligned}
& \bar{w}_{n}(\zeta, q)=\sqrt{\frac{2}{\pi}} \frac{1}{T^{2}} \frac{(-1)^{n}-1}{\lambda_{n}}\left[\frac{1}{q+q_{4, n}(\zeta)}\right. \\
& -\left[\left\{\lambda_{2} q^{3}+\left(\lambda_{1}+\lambda_{4}\left(\zeta^{2}+\lambda_{n}^{2}\right)+\lambda_{2} \Omega+\lambda_{4} \varepsilon\right) q^{2}+\left(\lambda_{3}\left(\zeta^{2}+\lambda_{n}^{2}\right)+\lambda_{1} \Omega+\lambda_{3} \varepsilon\right) q\right\}\right. \\
& /\left\{\left(\lambda_{2} q^{3}+\left(\lambda_{1}+\lambda_{4}\left(\zeta^{2}+\lambda_{n}^{2}\right)+\lambda_{2} \Omega+\lambda_{4} \varepsilon\right) q^{2}+\left(1+\lambda_{3}\left(\zeta^{2}+\lambda_{n}^{2}\right)+\lambda_{1} \Omega+\lambda_{3} \varepsilon\right) q\right.\right. \\
& \left.\left.\left.+\left(\zeta^{2}+\lambda_{n}^{2}\right)+\Omega+\varepsilon\right)\left(q+q_{4, n}(\zeta)\right)\right\}\right] \times \frac{1}{q^{3}}\left(1+2 \sum_{p=1}^{\infty}(-1)^{p} \exp (-p T q)\right) \\
& \bar{w}_{n}(\zeta, q)=2 \sqrt{\frac{2}{\pi}} \frac{1}{T^{2}} \frac{(-1)^{n}-1}{\lambda_{n}}\left[\frac{1}{q+q_{4, n}(\zeta)}\right. \\
& \left.-\frac{1}{\lambda_{2}} \frac{\lambda_{2} q^{3}+\left(\lambda_{1}+\lambda_{4}\left(\zeta^{2}+\lambda_{n}^{2}\right)+\lambda_{2} \Omega+\lambda_{4} \varepsilon\right) q^{2}+\left(\lambda_{3}\left(\zeta^{2}+\lambda_{n}^{2}\right)+\lambda_{1} \Omega+\lambda_{3} \varepsilon\right) q}{\left(q-q_{1, n}(\zeta)\right)\left(q-q_{2, n}(\zeta)\right)\left(q-q_{3, n}(\zeta)\right)\left(q+q_{4, n}(\zeta)\right)}\right] \\
& \times \frac{1}{q^{3}}\left(1+2 \sum_{p=1}^{\infty}(-1)^{p} \exp (-p T q)\right) .
\end{aligned}
$$

Writing the above equation as

$$
\begin{aligned}
\bar{w}_{n}(\zeta, q)= & 2 \sqrt{\frac{2}{\pi}} \frac{1}{T^{2}} \frac{(-1)^{n}-1}{\lambda_{n}}\left(\frac{1}{q+q_{4, n}(\zeta)}-\frac{1}{\lambda_{2}}\left[\frac{\varphi_{1, n}(\zeta)}{q-q_{1, n}(\zeta)}+\frac{\varphi_{2, n}(\zeta)}{q-q_{2, n}(\zeta)}\right.\right. \\
& \left.\left.+\frac{\varphi_{3, n}(\zeta)}{q-q_{3, n}(\zeta)}+\frac{\varphi_{4, n}(\zeta)}{q+q_{4, n}(\zeta)}\right]\right) \times \frac{1}{q^{3}}\left(1+2 \sum_{p=1}^{\infty}(-1)^{p} \exp (-p T q)\right)
\end{aligned}
$$

where

$$
\begin{gathered}
\varphi_{1, n}(\zeta)=\frac{\phi_{1, n}(\zeta)}{\left(q_{1, n}(\zeta)-q_{2, n}(\zeta)\right)\left(q_{1, n}(\zeta)-q_{3, n}(\zeta)\right)\left(q_{1, n}(\zeta)+q_{4, n}(\zeta)\right)} \\
\varphi_{2, n}(\zeta)=\frac{\phi_{2, n}(\zeta)}{\left(q_{2, n}(\zeta)-q_{1, n}(\zeta)\right)\left(q_{2, n}(\zeta)-q_{3, n}(\zeta)\right)\left(q_{2, n}(\zeta)+q_{4, n}(\zeta)\right)} \\
\varphi_{3, n}(\zeta)=\frac{\phi_{3, n}(\zeta)}{\left(q_{3, n}(\zeta)-q_{1, n}(\zeta)\right)\left(q_{3, n}(\zeta)-q_{2, n}(\zeta)\right)\left(q_{3, n}(\zeta)+q_{4, n}(\zeta)\right)} \\
\varphi_{4, n}(\zeta)=\frac{\phi_{4, n}(\zeta)}{\left(q_{4, n}(\zeta)-q_{1, n}(\zeta)\right)\left(q_{4, n}(\zeta)-q_{2, n}(\zeta)\right)\left(q_{4, n}(\zeta)-q_{3, n}(\zeta)\right)} \\
q_{4, n}(\zeta)=\zeta^{2}+\lambda_{n}^{2}+\Omega+\varepsilon \\
q_{i, n}(\zeta)=s_{i, n}(\zeta)-\frac{\lambda_{1}+\lambda_{4}\left(\zeta^{2}+\lambda_{n}^{2}\right)+\lambda_{2} \Omega+\lambda_{4} \varepsilon}{3 \lambda_{2}^{2}}
\end{gathered}
$$




$$
\begin{aligned}
\phi_{i, n}(\zeta)= & \lambda_{2} q_{i, n}^{3}(\zeta)+\left(\lambda_{1}+\lambda_{4}\left(\zeta^{2}+\lambda_{n}^{2}\right)+\lambda_{2} \Omega+\lambda_{4} \varepsilon\right) q_{i, n}^{2}(\zeta) \\
& +\left(\lambda_{3}\left(\zeta^{2}+\lambda_{n}^{2}\right) \lambda_{1} \Omega+\lambda_{3} \varepsilon\right) q_{i, n}(\zeta) \text { for } n=1,2,3 . \\
\phi_{4 . n}(\zeta)= & -\lambda_{2} q_{4, n}^{3}(\zeta)+\left(\lambda_{1}+v \lambda_{4}\left(\zeta^{2}+\lambda_{n}^{2}\right)+\lambda_{2} \Omega+\lambda_{4} \varepsilon\right) q_{4, n}^{2}(\zeta) \\
& -\left(\lambda_{3}\left(\zeta^{2}+\lambda_{n}^{2}\right) \lambda_{1} \Omega+\lambda_{3} \varepsilon\right) q_{4, n}(\zeta),
\end{aligned}
$$

In the above relations

$$
\begin{aligned}
s_{1, n}(\zeta)= & \left(-\frac{\beta_{1, n}(\zeta)}{2}+\sqrt{\frac{\beta_{1, n}^{2}(\zeta)}{4}+\frac{\alpha_{1, n}^{3}(\zeta)}{27}}\right)^{1 / 3} \\
& +\left(-\frac{\beta_{1, n}(\zeta)}{2}-\sqrt{\frac{\beta_{1, n}^{2}(\zeta)}{4}+\frac{\alpha_{1, n}^{3}(\zeta)}{27}}\right)^{1 / 3}, \\
s_{2, n}(\zeta)= & Z\left(-\frac{\beta_{1, n}(\zeta)}{2}+\sqrt{\frac{\beta_{1, n}^{2}(\zeta)}{4}+\frac{\alpha_{1, n}^{3}(\zeta)}{27}}\right)^{1 / 3} \\
& +Z^{2}\left(-\frac{\beta_{1, n}(\zeta)}{2}-\sqrt{\frac{\beta_{1, n}^{2}(\zeta)}{4}+\frac{\alpha_{1, n}^{3}(\zeta)}{27}}\right)^{1 / 3}, \\
s_{3, n}(\zeta)= & Z^{2}\left(-\frac{\beta_{1, n}(\zeta)}{2}+\sqrt{\frac{\beta_{1, n}^{2}(\zeta)}{4}+\frac{\alpha_{1, n}^{3}(\zeta)}{27}}\right)^{1 / 3} \\
& +Z\left(-\frac{\beta_{1, n}(\zeta)}{2}-\sqrt{\frac{\beta_{1, n}^{2}(\zeta)}{4}+\frac{\alpha_{1, n}^{3}(\zeta)}{27}}\right)^{1 / 3},
\end{aligned}
$$

where

$$
\begin{gathered}
\alpha_{1, n}(\zeta)=\frac{1+v \lambda_{3}\left(\zeta^{2}+\lambda_{n}^{2}\right)+\lambda_{1} \Omega+\lambda_{3} \varepsilon}{\lambda_{2}}-\frac{\left(\lambda_{1}+v \lambda_{4}\left(\zeta^{2}+\lambda_{n}^{2}\right)+\lambda_{2} \Omega+\lambda_{4} \varepsilon\right)^{2}}{3 \lambda_{2}^{2}}, \\
\beta_{1, n}(\zeta)=\frac{v\left(\zeta^{2}+\lambda_{n}^{2}\right)+\Omega+\varepsilon}{\lambda_{2}}+2 \frac{\left(1+v \lambda_{3}\left(\zeta^{2}+\lambda_{n}^{2}\right)+\lambda_{1} \Omega+\lambda_{3} \varepsilon\right)^{3}}{27 \lambda_{2}^{3}} \\
-\frac{1}{3 \lambda_{2}^{2}}\left\{\left(\lambda_{1}+v \lambda_{4}\left(\zeta^{2}+\lambda_{n}^{2}\right)+\lambda_{2} \Omega+\lambda_{4} \varepsilon\right)\left(1+v \lambda_{3}\left(\zeta^{2}+\lambda_{n}^{2}\right)+\lambda_{1} \Omega+\lambda_{3} \varepsilon\right)\right\} \\
Z=\frac{-1+i \sqrt{3}}{2} .
\end{gathered}
$$

To solve Equation (27), we use the formula

$$
L^{-1}\left(\frac{1}{q^{3}\left(q-q_{1, n}(\zeta)\right)}\right)=\frac{\exp \left(q_{1, n}(\zeta) t\right)-\frac{1}{2} q_{1, n}^{2}(\zeta) t^{2}-q_{1, n}(\zeta) t-1}{q_{1, n}^{3}(\zeta)} .
$$

Inversion of Equation (26) by means of the Laplace transform and Fourier cosine and sine transforms, and using Equation (42), we obtain 


$$
\begin{aligned}
& w(x, y, t)=4 \sum_{n=1}^{\infty} \sqrt{\frac{2}{\pi}} \sin \left(\lambda_{n} y\right) \int_{0}^{\infty} B_{n}(\zeta) \cos (\zeta x) \\
& \times\left[\frac { 1 } { q _ { 4 , n } ^ { 3 } ( \zeta ) } \left\{\left(\exp \left(-q_{4, n}(\zeta) t\right)-\frac{1}{2} q_{4, n}^{2}(\zeta) t^{2}-q_{4, n}(\zeta) t-1\right) H(t)\right.\right. \\
& +2 \sum_{p=1}^{\infty}(-1)^{p} \times\left(\exp \left(-q_{4, n}(\zeta)(t-p T)\right)\right. \\
& \left.\left.\left.-\frac{1}{2} q_{4, n}^{2}(\zeta)(t-p T)^{2}-q_{4, n}(\zeta)(t-p T)-1\right) H_{p T}(t)\right\}\right] \mathrm{d} \zeta \\
& -2 \sum_{n=1}^{\infty} \sqrt{\frac{2}{\pi}} \sin \left(\lambda_{n} y\right) \int_{0}^{\infty} A_{n}(\zeta) \cos (\zeta x) \\
& \times\left[\left\{\left[\varphi_{1, n}(\zeta) \frac{\exp \left(q_{1, n}(\zeta) t\right)-\frac{1}{2} q_{1, n}^{2}(\zeta) t^{2}-q_{1, n}(\zeta) t-1}{q_{1, n}^{3}(\zeta)}\right.\right.\right. \\
& +\varphi_{2, n}(\zeta) \frac{\exp \left(q_{2, n}(\zeta) t\right)-\frac{1}{2} q_{2, n}^{2}(\zeta) t^{2}-q_{2, n}(\zeta) t-1}{q_{2, n}^{3}(\zeta)} \\
& +\varphi_{3, n}(\zeta) \frac{\exp \left(q_{3, n}(\zeta) t\right)-\frac{1}{2} q_{3, n}^{2}(\zeta) t^{2}-q_{3, n}(\zeta) t-1}{q_{3, n}^{3}(\zeta)} \\
& \left.\left.-\varphi_{4, n}(\zeta) \frac{\exp \left(-q_{4, n}(\zeta) t\right)-\frac{1}{2} q_{4, n}^{2}(\zeta) t^{2}+q_{4, n}(\zeta) t-1}{q_{4, n}^{3}(\zeta)}\right)\right\} H(t)+2 \sum_{p=1}^{\infty}(-1)^{p} \\
& \times\left\{\left(\varphi_{1, n}(\zeta) \frac{\exp \left(q_{1, n}(\zeta)(t-p T)\right)-\frac{1}{2} q_{1, n}^{2}(\zeta)(t-p T)^{2}-q_{1, n}(\zeta)(t-p T)-1}{q_{1, n}^{3}(\zeta)}\right.\right. \\
& +\varphi_{2, n}(\zeta) \frac{\exp \left(q_{2, n}(\zeta)(t-p T)\right)-\frac{1}{2} q_{2, n}^{2}(\zeta)(t-p T)^{2}-q_{2, n}(\zeta)(t-p T)-1}{q_{2, n}^{3}(\zeta)} \\
& +\varphi_{3, n}(\zeta) \frac{\exp \left(q_{3, n}(\zeta)(t-p T)\right)-\frac{1}{2} q_{3, n}^{2}(\zeta)(t-p T)^{2}-q_{3, n}(\zeta)(t-p T)-1}{q_{3, n}^{3}(\zeta)} \\
& \left.\left.-\varphi_{4, n}(\zeta) \frac{\exp \left(-q_{4, n}(\zeta)(t-p T)\right)-\frac{1}{2} q_{4, n}^{2}(\zeta)(t-p T)^{2}+q_{4, n}(\zeta)(t-p T)-1}{q_{4, n}^{3}(\zeta)}\right)\right\} \\
& \left.\times H_{p T}(t)\right] \mathrm{d} \zeta
\end{aligned}
$$

where

$$
A_{n}(\zeta)=\sqrt{\frac{2}{\pi}} \frac{1}{T^{2}} \frac{(-1)^{n}-1}{\lambda_{n} \lambda_{2}}, B_{n}(\zeta)=\sqrt{\frac{2}{\pi}} \frac{1}{T^{2}} \frac{(-1)^{n}-1}{\lambda_{n}} .
$$


The first part of Equation (43) gives solution for Newtonian fluid while the second part gives the corresponding non-Newtonian contribution.

The transient part of velocity for

$$
\begin{aligned}
& \operatorname{Re}\left(q_{1, n}(\zeta)\right), \operatorname{Re}\left(q_{2, n}(\zeta)\right), \operatorname{Re}\left(q_{3, n}(\zeta)\right)<0, \operatorname{Re}\left(q_{4, n}(\zeta)\right)>0 \text { is } \\
& w_{t}(x, y, t)=4 \sum_{n=1}^{\infty} \sqrt{\frac{2}{\pi}} \sin \left(\lambda_{n} y\right) \int_{0}^{\infty} B_{n}(\zeta) \cos (\zeta x) \\
& \times\left[\frac{1}{q_{4, n}^{3}(\zeta)}\left\{H(t)+2 \sum_{p=1}^{\infty}(-1)^{p} H_{p T}(t)\right\} \mathrm{d} \zeta\right. \\
&-2 \sum_{n=1}^{\infty} \sqrt{\frac{2}{\pi}} \sin \left(\lambda_{n} y\right) \int_{0}^{\infty} A_{n}(\zeta) \cos (\zeta x)
\end{aligned}
$$$$
\times\left[\left\{\frac{\varphi_{1, n}(\zeta)}{q_{1, n}^{3}(\zeta)}\left(H(t)+2 \sum_{p=1}^{\infty}(-1)^{p} \exp \left(q_{1, n}(\zeta) p T\right) H_{p T}(t)\right)\right.\right.
$$$$
+\varphi_{2, n}(\zeta) \frac{\exp \left(q_{2, n}(\zeta) t\right)-\frac{1}{2} q_{2, n}^{2}(\zeta) t^{2}-q_{2, n}(\zeta) t-1}{q_{2, n}^{3}(\zeta)}
$$$$
+\varphi_{3, n}(\zeta) \frac{\exp \left(q_{3, n}(\zeta) t\right)-\frac{1}{2} q_{3, n}^{2}(\zeta) t^{2}-q_{3, n}(\zeta) t-1}{q_{3, n}^{3}(\zeta)}
$$$$
\left.\left.-\varphi_{4, n}(\zeta) \frac{\exp \left(-q_{4, n}(\zeta) t\right)-\frac{1}{2} q_{4, n}^{2}(\zeta) t^{2}+q_{4, n}(\zeta) t-1}{q_{4, n}^{3}(\zeta)}\right)\right\} H(t)+2 \sum_{p=1}^{\infty}(-1)^{p}
$$$$
\times\left\{\left(\varphi_{1, n}(\zeta) \frac{\exp \left(q_{1, n}(\zeta)(t-p T)\right)-\frac{1}{2} q_{1, n}^{2}(\zeta)(t-p T)^{2}-q_{1, n}(\zeta)(t-p T)-1}{q_{4, n}^{3}(\zeta)}\right.\right.
$$$$
+\varphi_{2, n}(\zeta) \frac{\exp \left(q_{2, n}(\zeta)(t-p T)\right)-\frac{1}{2} q_{2, n}^{2}(\zeta)(t-p T)^{2}-q_{2, n}(\zeta)(t-p T)-1}{q_{2, n}^{3}(\zeta)}
$$$$
+\varphi_{3, n}(\zeta) \frac{\exp \left(q_{3, n}(\zeta)(t-p T)\right)-\frac{1}{2} q_{3, n}^{2}(\zeta)(t-p T)^{2}-q_{3, n}(\zeta)(t-p T)-1}{q_{3, n}^{3}(\zeta)}
$$$$
\left.\left.-\varphi_{4, n}(\zeta) \frac{\exp \left(-q_{4, n}(\zeta)(t-p T)\right)-\frac{1}{2} q_{4, n}^{2}(\zeta)(t-p T)^{2}+q_{4, n}(\zeta)(t-p T)-1}{q_{4, n}^{3}(\zeta)}\right)\right\}
$$

$\left.\times H_{p T}(t)\right] \mathrm{d} \zeta$,

while the steady state part is given by 


$$
\begin{aligned}
& w_{s}(x, y, t)=4 \sum_{n=1}^{\infty} \sqrt{\frac{2}{\pi}} \sin \left(\lambda_{n} y\right) \int_{0}^{\infty} B_{n}(\zeta) \cos (\zeta x) \\
& \times\left[\frac { 1 } { q _ { 4 , n } ^ { 3 } ( \zeta ) } \left\{\left(-\frac{1}{2} q_{4, n}^{2}(\zeta) t^{2}-q_{4, n}(\zeta) t-1\right) H(t)\right.\right. \\
& \left.\left.+2 \sum_{p=1}^{\infty}(-1)^{p}\left(-\frac{1}{2} q_{4, n}^{2}(\zeta)(t-p T)^{2}+q_{4, n}(\zeta)(t-p T)-1\right) H_{p T}(t)\right\}\right] \mathrm{d} \zeta \\
& -2 \sum_{n=1}^{\infty} \sqrt{\frac{2}{\pi}} \sin \left(\lambda_{n} y\right) \int_{0}^{\infty} A_{n}(\zeta) \cos (\zeta x)\left[\left\{\left(\varphi_{1, n}(\zeta) \frac{-\frac{1}{2} q_{1, n}^{2}(\zeta) t^{2}-q_{1, n}(\zeta) t-1}{q_{1, n}^{3}(\zeta)}\right.\right.\right. \\
& +\varphi_{2, n}(\zeta) \frac{-\frac{1}{2} q_{2, n}^{2}(\zeta) t^{2}-q_{2, n}(\zeta) t-1}{q_{2, n}^{3}(\zeta)}+\varphi_{3, n}(\zeta) \frac{-\frac{1}{2} q_{3, n}^{2}(\zeta) t^{2}-q_{3, n}(\zeta) t-1}{q_{3, n}^{3}(\zeta)} \\
& \left.\left.-\varphi_{4, n}(\zeta) \frac{-\frac{1}{2} q_{4, n}^{2}(\zeta) t^{2}+q_{4, n}(\zeta) t-1}{q_{4, n}^{3}(\zeta)}\right)\right\} H(t) \\
& +2 \sum_{p=1}^{\infty}(-1)^{p}\left\{\left(\varphi_{1, n}(\zeta) \frac{-\frac{1}{2} q_{1, n}^{2}(\zeta)(t-p T)^{2}-q_{1, n}(\zeta)(t-p T)-1}{q_{1, n}^{3}(\zeta)}\right.\right. \\
& +\varphi_{2, n}(\zeta) \frac{-\frac{1}{2} q_{2, n}^{2}(\zeta)(t-p T)^{2}-q_{2, n}(\zeta)(t-p T)-1}{q_{2, n}^{3}(\zeta)} \\
& +\varphi_{3, n}(\zeta) \frac{-\frac{1}{2} q_{3, n}^{2}(\zeta)(t-p T)^{2}-q_{3, n}(\zeta)(t-p T)-1}{q_{3, n}^{3}(\zeta)} \\
& \left.\left.\left.-\varphi_{4, n}(\zeta) \frac{-\frac{1}{2} q_{4, n}^{2}(\zeta)(t-p T)^{2}+q_{4, n}(\zeta)(t-p T)-1}{q_{4, n}^{3}(\zeta)}\right)\right\} H_{p T}(t)\right] \mathrm{d} \zeta .
\end{aligned}
$$

\section{Calculation of Tangential Stresses}

To obtain the expressions for the shear stresses $\tau_{1}(x, y, t)$ and $\tau_{2}(x, y, t)$, applying the Laplace transform to Equations (11) and (12), we have the expressions

$$
\begin{aligned}
& \bar{\tau}_{1}(x, y, q)=\frac{1+\lambda_{3} q+\lambda_{4} q^{2}}{1+\lambda_{1} q+\lambda_{2} q^{2}} \frac{\partial \bar{w}(x, y, q)}{\partial x}, \\
& \bar{\tau}_{2}(x, y, q)=\frac{1+\lambda_{3} q+\lambda_{4} q^{2}}{1+\lambda_{1} q+\lambda_{2} q^{2}} \frac{\partial \bar{w}(x, y, q)}{\partial y},
\end{aligned}
$$

From Equation (26) with inverse Fourier cosine and sine transforms, we have

$$
\begin{aligned}
\bar{w}(x, y, q)= & 4 \sum_{n=1}^{\infty} \sqrt{\frac{2}{\pi}} A_{n}(\zeta) \sin \left(\lambda_{n} y\right) \int_{0}^{\infty} \cos (\zeta x) \\
& \times \frac{1}{\left(q-q_{1, n}(\zeta)\right)\left(q-q_{2, n}(\zeta)\right)\left(q-q_{3, n}(\zeta)\right)} \\
& \times \frac{1}{q^{3}}\left(1+2 \sum_{p=1}^{\infty}(-1)^{p} \exp (-p T q)\right) \mathrm{d} \zeta .
\end{aligned}
$$


Using Equation (48) in Equation (47), we have

$$
\begin{aligned}
\bar{\tau}_{1}(x, y, q)= & -4 \sum_{n=1}^{\infty} \sqrt{\frac{2}{\pi}} A_{n}(\zeta) \sin \left(\lambda_{n} y\right) \int_{0}^{\infty} \frac{\zeta \sin (\zeta x)\left(1+\lambda_{3} q+\lambda_{4} q^{2}\right)}{1+\lambda_{1} q+\lambda_{2} q^{2}} \\
& \times \frac{1}{\left(q-q_{1, n}(\zeta)\right)\left(q-q_{2, n}(\zeta)\right)\left(q-q_{3, n}(\zeta)\right)} \\
& \times \frac{1}{q^{3}}\left[1+2 \sum_{p=1}^{\infty}(-1)^{p} \exp (-p T q)\right] \mathrm{d} \zeta, \\
\bar{\tau}_{2}(x, y, q)= & 4 \sum_{n=1}^{\infty} \sqrt{\frac{2}{\pi}} \lambda_{n} A_{n}(\zeta) \cos \left(\lambda_{n} y\right) \int_{0}^{\infty} \frac{\cos (\zeta x)\left(1+\lambda_{3} q+\lambda_{4} q^{2}\right)}{1+\lambda_{1} q+\lambda_{2} q^{2}} \\
& \times \frac{1}{\left(q-q_{1, n}(\zeta)\right)\left(q-q_{2, n}(\zeta)\right)\left(q-q_{3, n}(\zeta)\right)} \\
& \times \frac{1}{q^{3}}\left[1+2 \sum_{p=1}^{\infty}(-1)^{p} \exp (-p T q)\right] \mathrm{d} \zeta .
\end{aligned}
$$

Let us take

$$
\bar{A}(q)=\frac{1+\lambda_{3} q+\lambda_{4} q^{2}}{1+\lambda_{1} q+\lambda_{2} q^{2}}
$$

and

$$
\bar{B}_{n}(\zeta, q)=\frac{1}{q^{3}\left(q-q_{1, n}(\zeta)\right)\left(q-q_{2, n}(\zeta)\right)\left(q-q_{3, n}(\zeta)\right)}
$$

Writing Equation (51) under the following equivalent form

$$
\bar{A}(q)=a_{2}+a_{3} \frac{q+a_{1}}{\left(q+a_{1}\right)^{2}-b_{1}^{2}}+a_{4} \frac{b_{1}}{\left(q+a_{1}\right)^{2}-b_{1}^{2}}
$$

where

$$
\begin{aligned}
& a_{1}=\frac{\lambda_{1}}{2 \lambda_{2}}, a_{2}=\frac{\lambda_{4}}{\lambda_{2}}, a_{3}=\frac{\lambda_{2} \lambda_{3}-\lambda_{1} \lambda_{4}}{\lambda_{2}^{2}}, \\
& a_{4}=\frac{2 \lambda_{2}\left(\lambda_{2}-\lambda_{4}\right)-\lambda_{1}\left(\lambda_{2} \lambda_{3}-\lambda_{1} \lambda_{4}\right)}{\lambda_{2}^{2} \sqrt{\lambda_{1}^{2}-4 \lambda_{2}}}, b_{1}=\frac{\sqrt{\lambda_{1}^{2}-4 \lambda_{2}}}{2 \lambda_{2}},
\end{aligned}
$$

and $\lambda_{1}^{2}-4 \lambda_{2}>0$.

Applying the inverse Laplace transform to Equation (53), we obtain

$$
A(t)=a_{2}+a_{3} \cosh \left(b_{1} t\right) \exp \left(-a_{1} t\right)+a_{4} \sinh \left(b_{1} t\right) \exp \left(-a_{1} t\right) .
$$

Now Equation (52) can also be written as

$$
\bar{B}_{n}(\zeta, q)=\frac{1}{q^{3}}\left(\frac{\psi_{1, n}(\zeta)}{q-q_{1, n}(\zeta)}+\frac{\psi_{2, n}(\zeta)}{q-q_{2, n}(\zeta)}+\frac{\psi_{3, n}(\zeta)}{q-q_{3, n}(\zeta)}\right)
$$

where

$$
\psi_{1, n}(\zeta)=\frac{1}{\left(q_{1, n}(\zeta)-q_{2, n}(\zeta)\right)\left(q_{1, n}(\zeta)-q_{3, n}(\zeta)\right)}
$$




$$
\begin{aligned}
& \psi_{2, n}(\zeta)=\frac{1}{\left(q_{2, n}(\zeta)-q_{1, n}(\zeta)\right)\left(q_{2, n}(\zeta)-q_{3, n}(\zeta)\right)} \\
& \psi_{3, n}(\zeta)=\frac{1}{\left(q_{3, n}(\zeta)-q_{1, n}(\zeta)\right)\left(q_{3, n}(\zeta)-q_{2, n}(\zeta)\right)}
\end{aligned}
$$

Applying the inverse Laplace transform to Equation (56), we obtain

$$
\begin{aligned}
B_{n}(\zeta)= & \psi_{1, n}(\zeta) \frac{\exp \left(q_{1, n}(\zeta) t\right)-\frac{1}{2} q_{1, n}^{2}(\zeta) t^{2}-q_{1, n}(\zeta) t-1}{q_{1, n}^{3}(\zeta)} \\
& +\psi_{2, n}(\zeta) \frac{\exp \left(q_{2, n}(\zeta) t\right)-\frac{1}{2} q_{2, n}^{2}(\zeta) t^{2}-q_{2, n}(\zeta) t-1}{q_{2, n}^{3}(\zeta)} \\
& +\psi_{3, n}(\zeta) \frac{\exp \left(q_{3, n}(\zeta) t\right)-\frac{1}{2} q_{3, n}^{2}(\zeta) t^{2}-q_{3, n}(\zeta) t-1}{q_{3, n}^{3}(\zeta)}
\end{aligned}
$$

Let

$$
\tau_{n}(\zeta, t)=(A * B)(t)=\int_{0}^{t} A(t-q) B_{n}(\zeta, q) \mathrm{d} q,
$$

Using Equations (55) and (60) in Equation (61), we obtain

$$
\begin{aligned}
\tau_{N}(\zeta, t)= & \int_{0}^{t}\left(a_{2}+a_{3} \cosh \left(b_{1}(t-q)\right) \exp \left(-a_{1}(t-q)\right)\right. \\
& \left.+a_{4} \sinh \left(b_{1}(t-q)\right) \exp \left(-a_{1}(t-q)\right)\right) \\
& \times\left(\psi_{1, n}(\zeta) \frac{\exp \left(q_{1, n}(\zeta) t\right)-\frac{1}{2} q_{1, n}^{2}(\zeta) t^{2}-q_{1, n}(\zeta) t-1}{q_{1, n}^{3}(\zeta)}\right. \\
& +\psi_{2, n}(\zeta) \frac{\exp \left(q_{2, n}(\zeta) t\right)-\frac{1}{2} q_{2, n}^{2}(\zeta) t^{2}-q_{2, n}(\zeta) t-1}{q_{2, n}^{3}(\zeta)} \\
& \left.+\psi_{3, n}(\zeta) \frac{\exp \left(q_{3, n}(\zeta) t\right)-\frac{1}{2} q_{3, n}^{2}(\zeta) t^{2}-q_{3, n}(\zeta) t-1}{q_{3, n}^{3}(\zeta)}\right) \mathrm{d} q .
\end{aligned}
$$

Inversion of Equations (49) and (50) by means of Laplace transform, using Equation (62) results in

$$
\begin{aligned}
\tau_{1}(x, y, t)= & -4 \sum_{n=1}^{\infty} \sqrt{\frac{2}{\pi}} A_{n}(\zeta) \sin \left(\lambda_{n} y\right) \int_{0}^{\infty} \zeta \sin (\zeta x) \\
& \times\left(\int_{0}^{t} \tau_{N}(\zeta, t) H(t)+2 \sum_{p=1}^{\infty}(-1)^{p} \int_{0}^{t-p T} \tau_{N}(\zeta, t-p T) H_{p T}(t)\right) \mathrm{d} \zeta, \\
\tau_{2}(x, y, t)= & 4 \sum_{n=1}^{\infty} \sqrt{\frac{2}{\pi}} \lambda_{n} A_{n}(\zeta) \cos \left(\lambda_{n} y\right) \int_{0}^{\infty} \cos (\zeta x) \\
& \times\left(\int_{0}^{t} \tau_{N}(\zeta, t) H(t)+2 \sum_{p=1}^{\infty}(-1)^{p} \int_{0}^{t-p T} \tau_{N}(\zeta, t-p T) H_{p T}(t)\right) \mathrm{d} \zeta .
\end{aligned}
$$

the shear stresses for the generalized Burgers' fluid. 


\section{Results and Discussion}

The present problem is concerned with an unsteady motion of generalized Burgers' fluid generated from rest induced by sawtooth pluses stress. The sawtsooths are of the form with quadratic edges. Laplace transform technique along with Fourier cosine and sine transforms have been used as mathematical tools in this order. The obtained expression for the velocity field has been written as the sum of Newtonian and non Newtonian contributions.

By using the numerical calculations and graphical illustrations, the following physical aspects of the fluid behavior have been analyzed.

Figure 1 is plotted the variation in time of the exciting force given on the bottom plate. It is observed that, the exciting force is increasing if the time $t$ increases, but is decreasing with the parameter $T$. By choosing the parameter $T$, we can modify the intensity of the stress force on the plate. Figure 2 represents the graphs of velocity profile for three values of time $t$ at different positions $x=0.1$, 0.6, 0.9 from the bottom plate for third harmonic verses $y$. It is seen that for small values of time $t$ the differences between the velocities are large and these differences decrease rapidly for large values of time. It is also to be noted that the magnitude of pulse oscillations decrease far from the bottom plate and the time after which the transient part can be neglected is decreasing. Moreover, this figure also reveals that the velocity profiles are in the same phase during the entire motion.

Figure 3 predicts the effect of magnetic field on the velocity profile verses $y$. From this figure, it is apparent that the effect of magnetic field is to enhance the velocity amplitudes. Figure 4 predicts the effect of pulse period on the velocity profile verses $y$. From this figure, it is apparent that the effect of pulse period

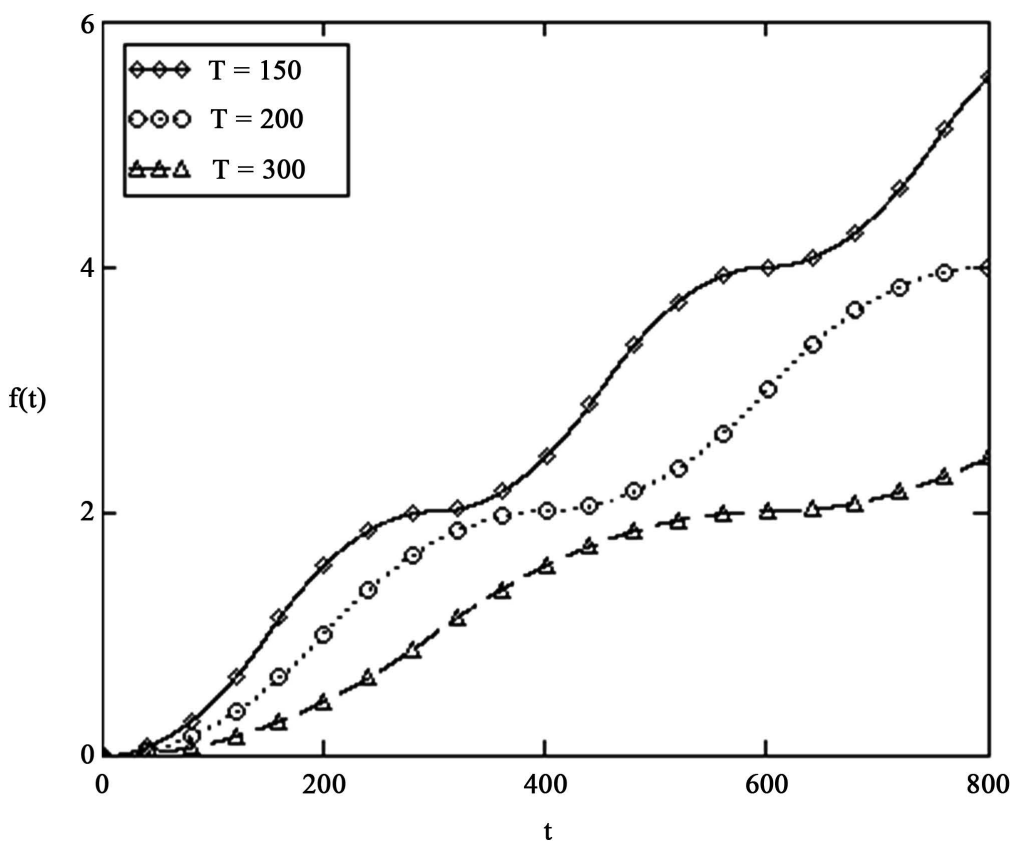

Figure 1. Evolution in time of the exciting function $f(t)$. 

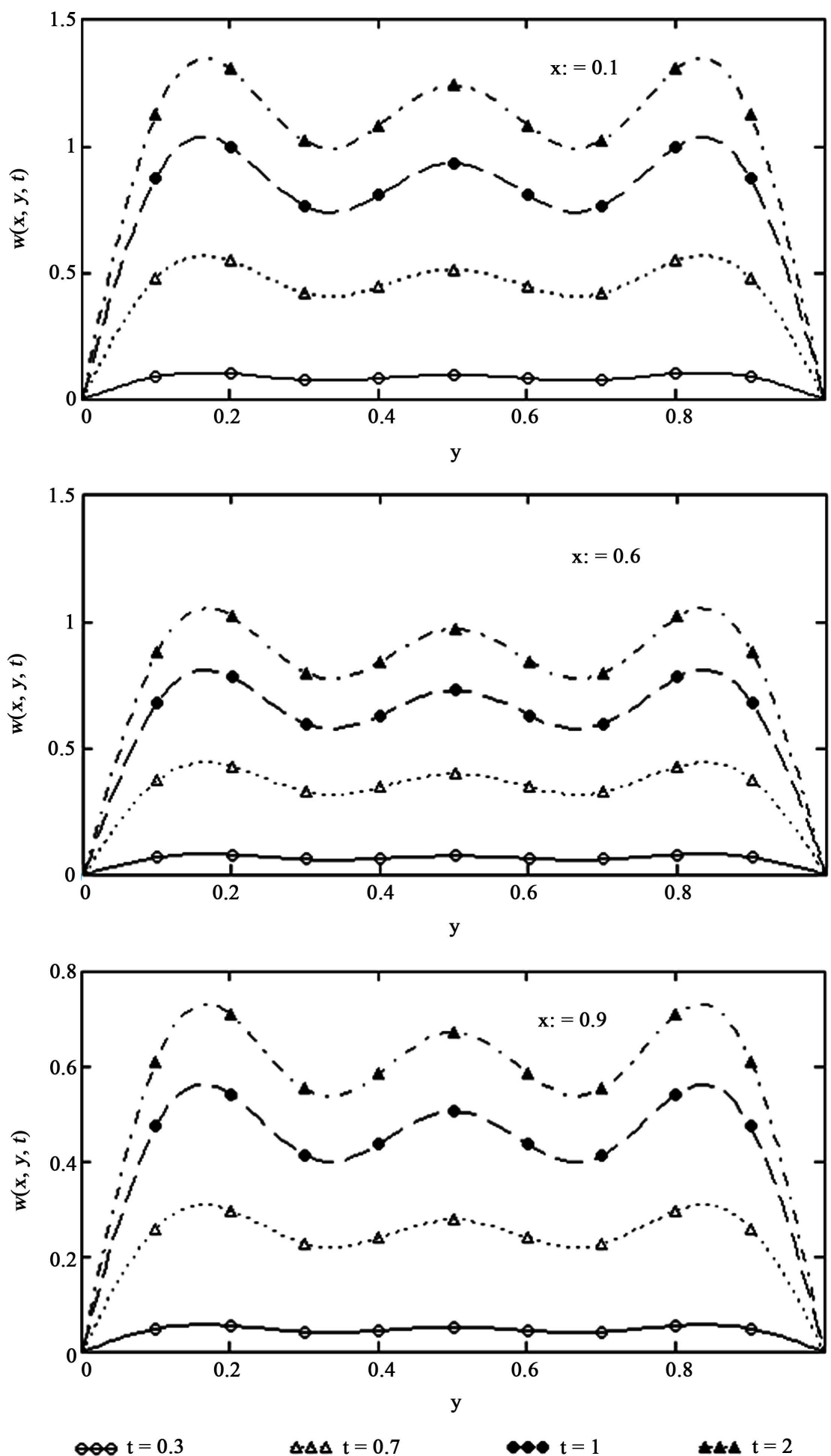

Figure 2. Velocity profiles for Generalized Burgers' fluid for different values of $t$ and $x$. Other parameters and values are taken as $\lambda_{1}=5, \lambda_{2}=7, \lambda_{3}=0.1, \lambda_{4}=3, T=\pi / 4, \Omega=1.7$, $\varepsilon=0.3$.

is opposite to that of magnetic field strength. Therefore, pulse period reduces the magnetic effect. 

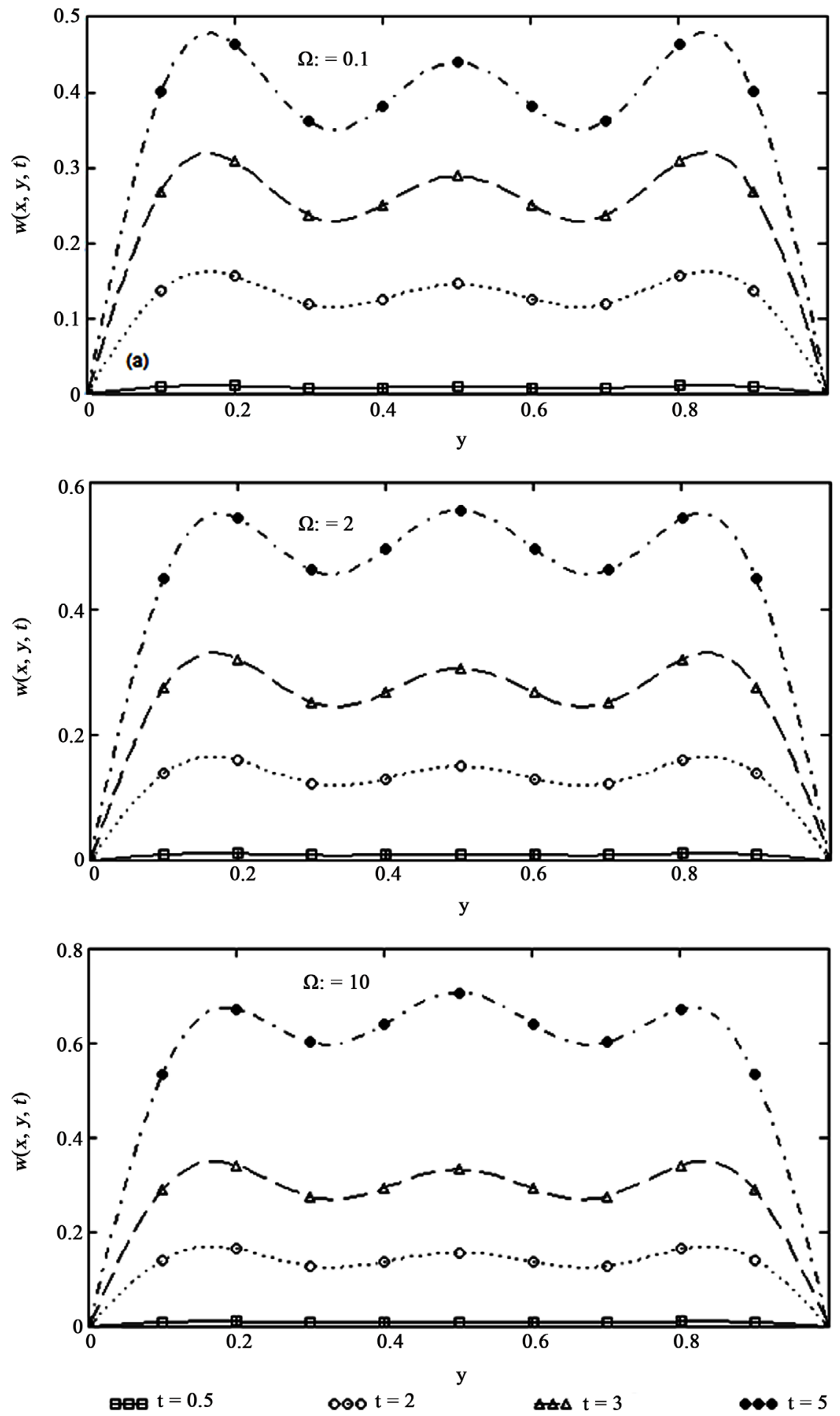

Figure 3. Velocity profiles for Generalized Burgers' fluid for different values of $\Omega$ and $t$. Other parameters and values are taken as $\lambda_{1}=5, \lambda_{2}=7, \lambda_{3}=0.1, \lambda_{4}=3, T=4, x=0.1$, $\varepsilon=0.3$.

In order to study the influence of various values of the material parameters $\lambda_{1}$, $\lambda_{2}, \lambda_{3}$ and $\lambda_{4}$, the diagrams of the velocity field from Figure 5 and Figure 6 

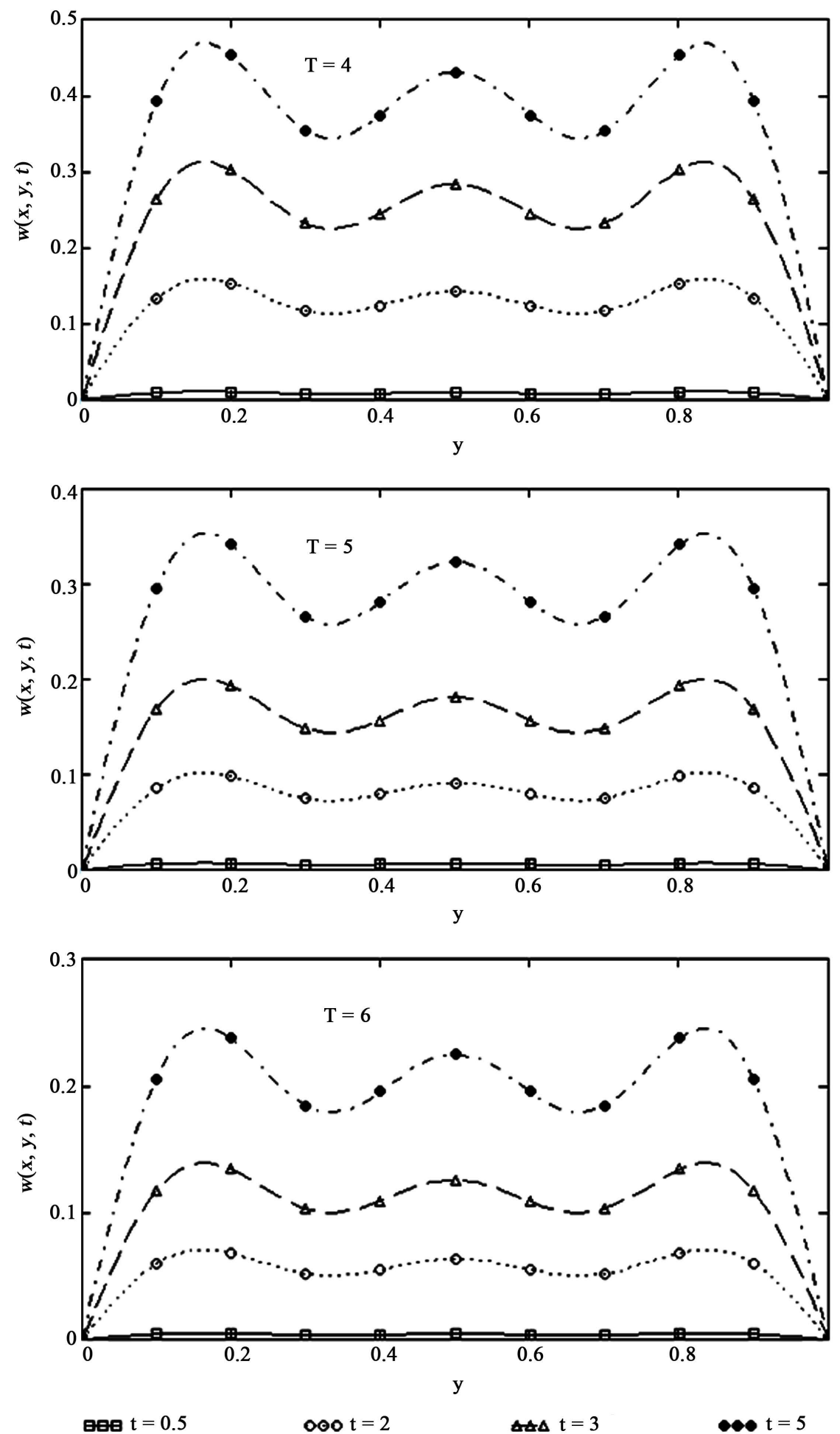

Figure 4. Velocity profiles for Generalized Burgers' fluid for different values of $T$ and $t$. Other parameters and values are taken as $\lambda_{1}=5, \lambda_{2}=7, \lambda_{3}=0.1, \lambda_{4}=3, T=4, x=0.2$, $\Omega=0.1, \varepsilon=0.3$.

are plotted. In these figures, we used the numerical values $x=0.1, y=0.1, T=4$, $\mathcal{E}=0.3, \Omega=0.1$. 

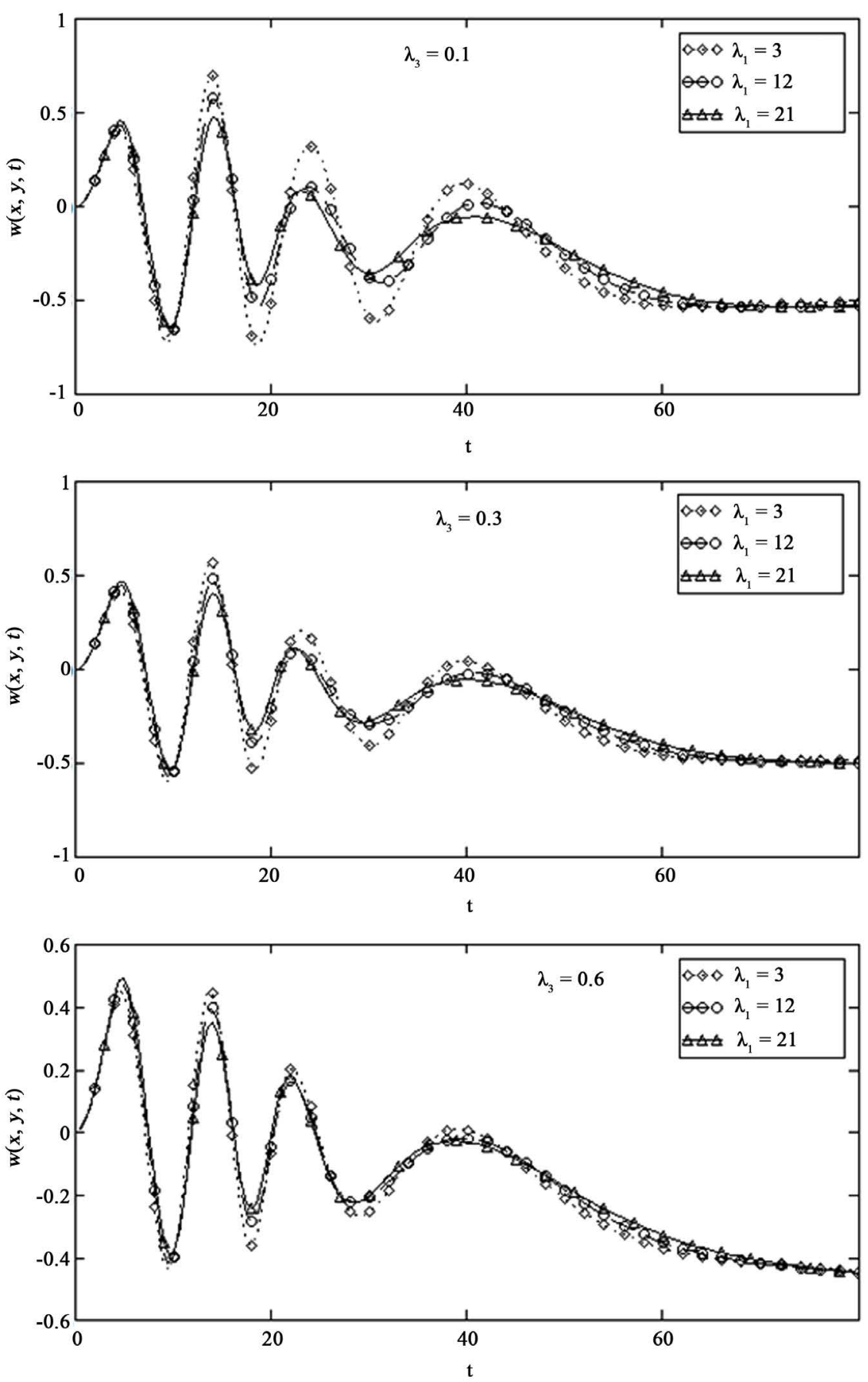

Figure 5. Velocity profiles for Generalized Burgers' fluid for different values of $\lambda_{1}$ and $\lambda_{3}$. Other parameters and values are taken as $\lambda_{2}=7, \lambda_{4}=3, y=0.1, x=0.1, \Omega=0.1$, $T=4, \varepsilon=0.3$.

In Figure 5, the parameters $\lambda_{1}$ and $\lambda_{3}$ are variables and parameters $\lambda_{2}$ and $\lambda_{4}$ are constant. It can be seen that, if the values of the parameter $\lambda_{3}$ increase, the fluid flows more slowly. It can also be noted that for the same values of the parameter $\lambda_{3}$, increasing of $\lambda_{1}$ values result in decreasing velocity of fluid flow (the velocity amplitudes decrease if the values of $\lambda_{1}$ increase). This figure also reveals 

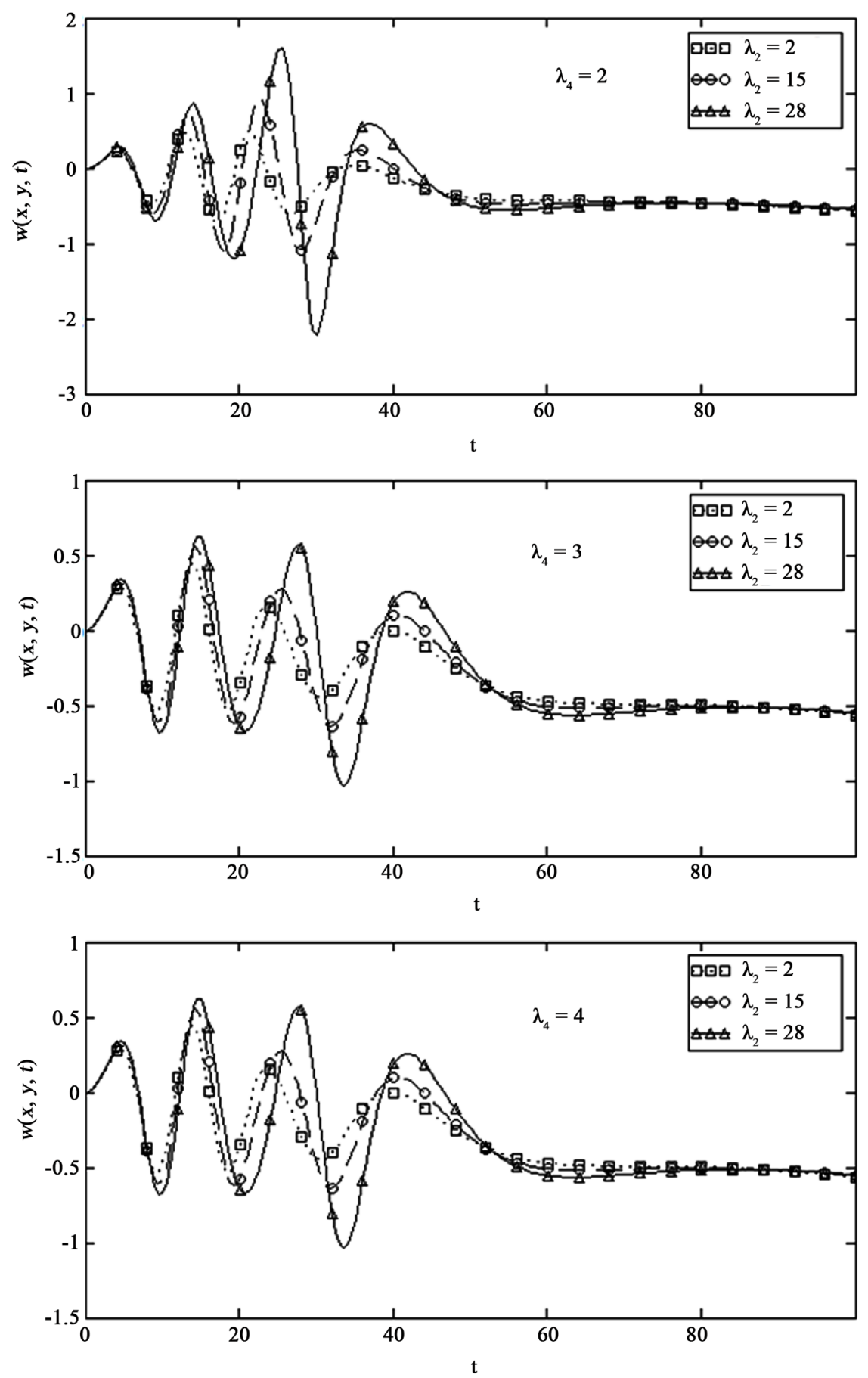

Figure 6. Velocity profiles for Generalized Burgers' fluid for different values of $\lambda_{2}$ and $\lambda_{4}$. Other parameters and values are taken as $\lambda_{1}=3, \lambda_{3}=0.1, y=0.1, x=0.1, \Omega=0.1$, $T=4, \varepsilon=0.3$.

that the velocity is a strong function of the Burgers' fluid parameter $\lambda_{3}$. Figure 6 corresponds to the variation of the parameters $\lambda_{2}$ and $\lambda_{4}$. There is no significant effect in the early period of the motion. The difference appears in the behavior of the fluid velocity, when compared with $\lambda_{3}$ that, velocity amplitudes increase if 
the parameter $\lambda_{2}$ increases. And in this case, for a constant value of the parameter $\lambda_{2}$, the increasing of $\lambda_{4}$, it is clear that, the fluid flows more slowly.

\section{Conclusions}

Here we obtained analytical solutions for the magnetohydrodynamic flow of a generalized Burgers' fluid between two parallel walls. The expressions for the velocity field and the corresponding tangential stresses induced by the sawtooth pulses stress are obtained by means of the Laplace and Fourier cosine and sine transforms in this order. The main findings are summarized as follows:

- The amplitude of pulse oscillation of velocity profile reduces far from the bottom plate.

- The magnitude of pulse oscillation of velocity profile increases from zero to maximum from the side walls till the middle of the channel.

- There is a time interval in which the velocity is oscillatory and then the oscillations of the velocity are attenuated.

- As distance between the walls increases, the magnitude of pulse oscillation of velocity profile also increases initially and then the oscillations of the velocity are attenuated.

- Increasing magnetic field and porosity of medium lead to increase the amplitude of pulse oscillation, while the effect of $T$ on the velocity profile is opposite.

- As $t$ increases, the pulse oscillation of velocity profile also increases verses $y$.

- As the values of the parameters $\lambda_{1}, \lambda_{3}$ and $\lambda_{4}$ increase, the fluid flows more slowly whereas behavior of $\lambda_{2}$ is opposite.

\section{Acknowledgements}

Authors are thankful to Prof. Dr. Jiayu Li, School of Mathematical Sciences, University of Science and Technology of China, Hefei, Anhui, China, for support and help.

\section{References}

[1] Zheng, L., Zhao, F. and Zhang, H. (2010) Exact Solutions for Generalized Maxwell Fluid Flow due to Oscillatory and Constantly Accelerating Plate. Nonlinear Analysis. Real World Applications, 11, 3744-3751. https://doi.org/10.1016/j.nonrwa.2010.02.004

[2] Ghosh, A.K. and Sana, P. (2099) On Hydromagnetic Flow of an Oldroyd-B Fluid near a Pulsating Plate. Acta Astronautica, 64, 272-280. https://doi.org/10.1016/j.actaastro.2008.07.016

[3] Akhtar, W., Fetecau, C., Tigoiu. V. and Fetecau, C. (2009) Flow of a Maxwell Fluid between Two Side Walls Induced by a Constantly Accelerating Plate. Zeitschrift für angewandte Mathematik und Physik, 60, 498-510. https://doi.org/10.1007/s00033-008-7129-8

[4] Fetecau, C., Nazar M. and Fetecau, F. (2009) Unsteady Flow of an Oldroyd-B Fluid Generated by a Constantly Accelerating Plate Between Two Side Walls Perpendicular to the Plate. International Journal of Non-Linear Mechanics, 44, 1039-1047. 
https://doi.org/10.1016/j.ijnonlinmec.2009.08.008

[5] Chakraborty, A. and Ray, T. (1980) Unsteady Magnetohydrodynamic Couette Flow between Two Plates When One of the Plates Is Subjected to Random Pulses. Journal of the Physical Society of Japan, 48, 1361-1364. https://doi.org/10.1143/JPSJ.48.1361

[6] Makar, M.N. (1987) Magnetohydrodynamic Flow between Two Plates When One of the Plates Is Subjected to Tooth Pulses. Acta Physica Polonica, 71, 995-999.

[7] Khan, M. and Zeeshan (2011) MHD Flow of an Oldroyd-B Fluid through a Porous Space Induced by Sawtooth Pulses. Chinese Physics Letters, 28, 84701-84704. https://doi.org/10.1088/0256-307X/28/8/084701

[8] Sohail, A., Vieru, D. and Imran, M.A. (2013) Influence of Side Walls on the Oscillating Motion of a Maxwell Fluid over an Infinite Plate. Mechanics, 9, 269-276. https://doi.org/10.5755/j01.mech.19.3.4665

[9] Ghosh, A.K. and Sana, P. (2009) On Hydromagnetic Channel Flow of an Oldroyd-B Fluid Induced by Rectified Sine Pulses. Computational and Applied Mathematics, 28, 365-395. https://doi.org/10.1590/S1807-03022009000300006

[10] Jamil, M. Fetecau, C. and Imran, M. (2011) Unsteady Helical Flows of Oldroyd-B Fluids. Communications in Nonlinear Science and Numerical Simulation, 16, 1378-1386. https://doi.org/10.1016/j.cnsns.2010.07.004

[11] Shahid, N. Rana, M. and Siddique, I. (2012) Exact Solution for Motion of an Oldroyd-B Fluid Over an Infinite Flat Plate That Applies an Oscillating Shear Stress to the Fluid. Boundary Value Problems, 2012, 48. https://doi.org/10.1186/1687-2770-2012-48

[12] Sultan, Q. Nazar, M. Imran, M. and Ali, U. (2014) Flow of Generalized Burgers Fluid between Parallel Walls Induced by Rectified Sine Pulses Stress. Boundary Value Problems, 2014, 152. https://doi.org/10.1186/1687-2770-2012-48

[13] Akhtar, W., Fetecau, C. and Awan, A.U. (2011) Exact Solutions for the Poiseuille Flow of a Generalized Maxwell Fluid Induced by Time-Dependent Shear Stress. ANZIAM Journal, 51, 416-429. https://doi.org/10.21914/anziamj.v51i0.1637

[14] Li, C., Zheng, L., Zhang, Y., Zhang, L. and Ma, X. (2012) Helical Flows of a Heated Generalized Oldroyd-B Fluid Subject to a Time-Dependent Shear Stress in Porous Medium. Communications in Nonlinear Science and Numerical Simulation, 17, 5026-5041. https://doi.org/10.1016/j.cnsns.2012.04.012

[15] Jamil, J. and Fetecau, F. (2010) Helical Flows of Maxwell Fluid between Coaxial Cylinders with Given Shear Stresses on the Boundary. Nonlinear Analysis. Real World Applications, 11, 4302-4311. https://doi.org/10.1016/j.nonrwa.2010.05.016

[16] Fetecau, C., Mahmood, A. and Jamil, M. (2010) Exact Solutions for the Flow of a Viscoelastic Fluid Induced by a Circular Cylinder Subject to a Time Dependent Shear Stress. Communications in Nonlinear Science and Numerical Simulation, 5, 3931-3938. https://doi.org/10.1016/j.cnsns.2010.01.012

[17] Qureshi, M.I., Imran, M., Athar, M. and Kamram, M. (2011) Analytic Solutions for the Unsteady Rotational Flow of an Oldroyd-B Fluid with Fractional Derivatives Induced by a Quadratic Time-Dependent Shear Stress. Applied and computational mathematics, 10, 484-497.

[18] Nazar, M., Shahid, F., Akram, M.S. and Sultan, Q. (2012) Flow on Oscillating Rectangular Duct for Maxwell Fluid. Applied Mathematics and Mechanics, 33, 717-730. https://doi.org/10.1007/s10483-012-1582-6

[19] Stehfest, H. (1970) Algorithm 368: Numerical Inversion of Laplace Transform. Communication of the ACM, 13, 47-49. https://doi.org/10.1145/361953.361969 